\section{Bioética e nutrição em cuidados paliativos oncológicos em adultos}

\author{
Bioethics and nutrition in adult patients with \\ cancer in palliative care
}

\section{${ }^{1}$ Instituto Nacional de Câncer, Rio de Janeiro, Brasil. 2 Universidade Federal de São João Del-Rei, Divinópolis, Brasil \\ Correspondência M. O. Benarroz \\ Instituto Nacional de Câncer. Rua Visconde de Santa Isabel 274, Rio de Janeiro, $R J$ 20560-120, Brasil. \\ monicabenarroz@hotmail.com}

\section{Abstract}

Cancer constitutes a major group of chronic diseases and is the second leading cause of death in the developed countries. Palliative care proposes to offer comprehensive support to control symptoms and improve quality of life for patients and their families. Nutrition is an important tool in palliative care, helping patients with their physical, psychological, and social issues and promoting comfort and quality of life. However, in the context of palliative care, nutritional support rarely achieves its role of fully recovering and assuring nutritional status. At this point, the nutritionist must consider the individual patient's needs, preferences, and eating habits, which are essential both for controlling symptoms and assuring satisfaction and comfort. The impossibility of conventionally applying established management and the development of a new perception of the patient often raise dilemmas for professional nutritionists.

Hospice Care; Quality of Life; Neoplasms; Bioethics; Public Health Nutrition
Monica de Oliveira Benarroz ${ }^{1}$

Giovanna Borges Damião Faillace 1

Leandro Augusto Barbosa 1,2

\section{Introdução}

As doenças crônicas são as principais causas de morte no mundo 1 , dentre as quais o câncer tem se destacado por seu crescimento em todos os continentes. Nos países desenvolvidos e em desenvolvimento, encontra-se como a segunda e a terceira causa de morte, respectivamente 2 .

Para os casos em que os indivíduos recebem o diagnóstico com a doença em estádio avançado, quando não há mais tratamento curativo, a Organização Mundial da Saúde (OMS) descreve um modelo de atenção à saúde cuja essência é minimizar os efeitos adversos ou complicações dos procedimentos médicos ${ }^{3}$. Seu objetivo é a qualidade de vida dos pacientes e de seus familiares 2 .

Esse tipo de assistência foi denominado de cuidados paliativos, definidos como uma modalidade que melhora a qualidade de vida dos pacientes - que enfrentam uma doença sem possibilidades de cura - e de suas famílias, por meio de prevenção e alívio do sofrimento, da identificação precoce, da avaliação impecável e do tratamento da dor e de outros sintomas físicos, psicossociais e espirituais ${ }^{2}$. Tais cuidados igualmente podem ser direcionados a pessoas que receberam tratamento curativo e aos sobreviventes do câncer que tiveram efeitos adversos tardios ou comorbidades relacionadas aos tratamentos recebidos 4 . 
A medicina ocidental tem enfatizado muito a qualidade de vida ${ }^{3}$, difícil de ser obtida e definida, pois é um conceito multidimensional, dinâmico e subjetivo ${ }^{5}$. Porém, é sabido que, assim como o bem-estar, está associada à saúde e não à doença. Segundo a OMS, qualidade de vida é a percepção do indivíduo em relação a sua posição na vida, no contexto da cultura e do sistema de valores em que vive, considerando seus objetivos, expectativas, padrões e interesses ${ }^{6}$. Levando-se em consideração esses conceitos, percebemos que, apesar da doença, é possível minimizar o sofrimento valendo-se de cuidados ativos 4 .

Com base no exposto, a nutrição também é um assunto pertinente aos cuidados paliativos e inclui a alimentação artificial (cateter ou ostomias) ${ }^{3}$. Considerada essencial para a existência humana, tem papel relevante na promoção de saúde e na prevenção de doenças 1,2 . Pode ser utilizada como tratamento quando visa a contribuir para reverter o estado metabólico e/ou nutricional mediante balanço energético, ajustes de micronutrientes, eletrólitos e reposição de alguma substância funcional 4,7,8

Portanto, a prática assistencial deve compreender o cuidado nutricional 9 , necessário em todos os estádios da doença e na estratégia terapêutica 4,7. Em cuidados paliativos, a nutrição tem especial papel preventivo, possibilitando meios e vias de alimentação, reduzindo os efeitos adversos provocados pelos tratamentos, retardando a síndrome anorexia-caquexia e ressignificando o alimento. Em adição, auxilia no controle de sintomas, mantém hidratação satisfatória, preserva o peso e a composição corporais 4,7,10.

\section{Cuidados paliativos: um outro olhar}

Os cuidados paliativos transcendem um modelo assistencial, pois descrevem uma abordagem holística 5,11. São pautados na humanização do atendimento mediante a capacitação de profissionais, familiares e/ou cuidadores para lidarem com o doente, no suporte terapêutico até o final da vida 2,12 , e em cuidados preventivos na dor, no controle de sinais e sintomas e nas perdas psicossociais 11,12. Essas ações não têm como objetivo esgotar as possibilidades de tratamento, como na medicina conservadora, a qual utiliza toda tecnologia médica para prolongar o processo de morrer, procedimento este caracterizado como futilidade ou obstinação terapêutica (distanásia) $13,14,15$.

Não é fácil individualizar a fase de tratamento paliativo no curso da evolução da doença porque os tratamentos ativos atualmente aplicados não trazem esperança real da cura do câncer 10. A so- brevida prevista é um dos critérios principais da definição das fases paliativas e terminais. Uma doença terminal é aquela que não responde a nenhum tratamento disponível, e pode-se racionalmente estimar, com grande probabilidade, um resultado fatal em um curto prazo baseado no melhor diagnóstico disponível 10.

A visão filosófica dos cuidados paliativos está pautada em princípios fundamentais: valoriza a vida e considera a morte como um processo natural; nem abrevia e nem prolonga a vida. Sua prática, entretanto, é um desafio que demanda assistência de uma equipe interdisciplinar treinada e capacitada ao atendimento humanizado $2,4,7,10,12$. Cada profissional integrado à equipe decidirá junto com a família e o próprio paciente qual será a melhor conduta 10,11,16.

\section{Bioética e cuidados paliativos}

A Bioética, como ciência da sobrevivência humana 17 e significância ética da vida 18 , levou aos profissionais de saúde a busca da reflexão compartilhada, complexa e interdisciplinar.

Os cuidados paliativos tratam freqüentemente o tema bioética, porquanto lidam com a dor, a perda, o sofrimento e a morte $4,12,13,19$. Nessa concepção, o paciente, ainda que sem possibilidade de cura ou em fase terminal, deve ser tratado com dignidade $4,13,15$.

O novo cenário de avanço tecnológico, o aumento da expectativa de vida, a integração entre perspectivas e a formação profissional mais humanizada, diferenciada, pautada na ética, na biologia e no respeito à vida 18 , buscam a dignidade humana e a qualidade de vida, adequando ações que envolvem a vida e o viver 17,20. Esses conceitos, inerentes ao ser humano, constam na Declaração Universal dos Direitos Humanos como sendo o fundamento da liberdade, da justiça e da paz no mundo 21 .

O paciente, como sujeito biopsicossocial e espiritual, demanda cuidado integral e humanizado na assistência ${ }^{20,22}$. O conhecimento técnicocientífico, respaldado nos pilares da bioética principialista (beneficência, não-maleficência, autonomia e justiça), aplicada na prática clínica 18,23 como norteadora de decisões, deve ser considerado em prol da qualidade de atendimento ao paciente.

O princípio bioético do respeito à autonomia é o início do direito do paciente de questionar seu tratamento e assegurar que o plano de cuidado esteja em conformidade com seu desejo 10,21. Muitas vezes, esse princípio é entendido como absoluto para as boas práticas dos cuidados paliativos, contudo nem sempre o paciente está 
apto a tomar decisões. Ainda assim, deverá ser beneficiado com os cuidados 15 .

No contexto da interdisciplinaridade, é imprescindível que haja interação de pessoas, com linguagem e objetivos comuns, reconhecimento de possíveis diferenças, domínio de conteúdos específicos e síntese das questões abordadas, por meio de troca de saberes e opiniões entre as competências 17 .

Em assuntos relacionados à alimentação e nutrição é comum enfrentar dilemas bioéticos 4,24, pois a alimentação está relacionada ao estilo de vida e bem-estar 11, a valores culturais 3,11 , ao prazer e à vida, envolvendo relações sociais e familiares 10,11, estando, ainda, inserida na cultura como símbolo de vitalidade. O nutricionista, no papel de membro da equipe interdisciplinar, deverá ter toda sua conduta registrada em prontuário, com a responsabilidade de identificar as necessidades de hidratação e nutricionais de cada paciente 4 . Contudo, como muitos serviços não usam sistema de documentação padronizado, apenas itens relevantes como a dor são registrados, resultando em uma documentação incompleta e inconsistente.

Assim, equipes interdisciplinares e multiprofissionais devem estabelecer documentação padrão para garantir qualidade e avaliação dos resultados de maneira efetiva 5 .

\section{Comunicação: fundamental no cuidado ao paciente}

A comunicação verbal adequada, como medida terapêutica eficaz, constitui uma das bases dos cuidados paliativos, além do relacionamento interpessoal, como essência do cuidado e da leitura de sinais não verbais, estabelecidos por uma relação de confiança entre o paciente, o profissional de saúde e a família e/ou cuidador 22,24.

O bom-humor, como forma de comunicação espontânea, e a alegria, com destaque para o otimismo, utilizados com discernimento e cautela, proporcionam a construção de relações terapêuticas, relacionando-se à filosofia dos cuidados paliativos e à dimensão do cuidado emocional 22 . Por sua vez, o desenvolvimento da habilidade da escuta é uma ferramenta importante que capacita o profissional ao aconselhamento mais eficaz ${ }^{3}$. Da mesma forma, a comunicação escrita merece destaque para melhor compreensão do paciente e adesão ao tratamento 25 .

A forma como as informações são transmitidas, por meio da comunicação verbal e não-verbal, refere-se à expressão de palavras, posturas e atitudes e requer especial atenção e cuidado para a escuta, a percepção, a compreensão, a identificação das necessidades e o planejamento das ações 22 . Em virtude da presença de diversos profissionais, a comunicação entre eles é essencial para oferecer um plano de cuidados consistentes tanto para o paciente quanto para seus familiares 10,24 .

No entanto, alguns estudos mostram que profissionais de saúde estão fechados à comunicação quando se sentem incapazes de oferecer ajuda prática ou quando percebem sua incapacidade em administrar a preocupação do paciente 25 .

\section{Cuidados paliativos e nutrição: um dilema bioético}

O câncer tem um forte impacto sobre a capacidade física, o bem-estar psicológico e a vida social dos pacientes, apresentando uma influência significativa no estado nutricional e na qualidade de vida $4,7,9,10,25,26,27$.

Os pacientes com a doença avançada cursam com sinais e sintomas como náuseas, vômitos, alteração no paladar, saciedade precoce 10,28, anorexia, caquexia, fraqueza e dispnéia 7,10,26,27,29. Esse quadro é de relevância clínica, uma vez que leva à menor ingestão de alimentos, resultando em efeitos consideráveis, na medida em que agrava o quadro clínico e prejudica a qualidade de vida 4,7,10,25,26,28.

A presença de sintomas como xerostomia (sensação subjetiva de boca seca) e disgeusia (alteração no paladar), bastante comuns nos pacientes em tratamento com opióides, quimioterapia e radioterapia 30 , também é freqüente na desnutrição 31,32. A xerostomia, apesar de pouco valorizada pelos médicos, tem implicações sérias, como maior dificuldade na deglutição e articulação de palavras; alteração do paladar; maior risco de infecções da mucosa bucal e cáries dentárias; alterações no sono e danos psicossociais 30,32. Problemas de saúde oral limitam a escolha e o consumo de alimentos, por isso têm um profundo impacto no estado nutricional 33 .

Outra queixa comum é a perda de peso, associada à ingestão insatisfatória de alimentos e à desnutrição 7,26,28,34. Diversos pacientes com câncer avançado passam pela experiência da perda de peso associada às mudanças de hábitos alimentares, sendo a anorexia um sintoma comumente referido por eles 34,35 . A anorexia per se reduz a ingestão de alimentos e promove a perda de peso 7,26,28. No momento em que o paciente percebe mudanças na sua auto-imagem, ocorre um forte impacto negativo, pois simboliza a aproximação da morte, a perda de autonomia, a fraqueza física e psicológica, refletindo diretamente na piora da sua qualidade de vida 25 . 
Existem diversas estratégias, incluindo intervenções nutricionais (suplementos) e farmacológicas (orexígenos) para deter ou reverter o quadro de perda de peso. Uma abordagem comum para direcionar a desnutrição e a perda de peso é o aumento de ingestão calórica com suplementos orais, para garantir os requerimentos diários em casos de baixa ingestão alimentar, independentemente dos motivos 7,10,26,33. Essa conduta pode ter menos riscos e menor custo que a nutrição artificial 36, entretanto não há precisão nos poucos estudos disponíveis acerca de seu monitoramento, freqüência, quantidade e eficácia 25,26,28,33

A importância das dietas enterais enriquecidas com os imunomoduladores (ômega-3, arginina e nucleotídeos) em pacientes com câncer avançado igualmente tem sido foco de discussões, principalmente quanto à questão da melhora da função imune, a redução da resposta inflamatória, a funcionalidade intestinal e a boa relação custobenefício 7,37.

A ausência na literatura científica de modelos de alimentação para pacientes com câncer avançado prejudica a compreensão dos hábitos alimentares dessa população e interfere no aconselhamento nutricional ${ }^{28}$. Situações de ingestão inadequada de alimentos podem ser identificadas e melhoradas por intermédio do aconselhamento. A eficácia da conduta depende da capacidade de intervir individualmente, adequando os procedimentos às necessidades e expectativas do paciente $7,10,26$.

A dor também é um sintoma que pode afetar o comportamento, causando irritabilidade, alterações de sono, de humor e de apetite ${ }^{38}$. Quando relacionada ao câncer, é um fenômeno complexo, multidimensional, composto por elementos sensoriais, afetivos, cognitivos e comportamentais 39 .

O objetivo do cuidado nutricional é assegurar a ingestão alimentar, conforme as necessidades e recomendações nutricionais, por meio da orientação da dieta, da avaliação e monitoramento do estado nutricional 7,9. Quando se trata de cuidados paliativos, no entanto, o foco principal é melhorar a qualidade de vida, através do controle de sintomas associados ao consumo de alimentos 4,10, adiando a perda de autonomia e qualidade de vida 7,10 . Nesse caso, dentre as leis de uma alimentação balanceada e completa 40 , a lei da adequação é priorizada por considerar as necessidades do indivíduo, suas preferências e seus hábitos alimentares fundamentais, tanto para o controle dos sintomas quanto para garantir satisfação (Tabela 1) 4,10.

Ainda que a proposta dos cuidados paliativos seja oferecer um sistema de apoio para ajudar os pacientes a viver tão ativos quanto possível até a morte ${ }^{2}$, em muitas situações o profissional de saúde depara com conflitos bioéticos 15, sobretudo ao lidar com a impossibilidade de alimentar ou hidratar o paciente, cuidados considerados básicos 4,10,35,41.

Membros das famílias associam alimentos com saúde e comumente relatam mais interesse em questões relativas à nutrição, se comparada com outras questões médicas complexas 24 .

Oferecer alimentos e líquidos ao moribundo é sinônimo de humanidade e compaixão. A alimentação é considerada um ícone associado à saúde e ao bem-estar, essencial à sobrevivência humana, com representatividades social e emocional, tornando-se importante no câncer avançado 4,11 . Sendo fonte de vida, a sua privação significa atentar contra a vida ${ }^{10}$. Não poder ou não conseguir se alimentar significa, portanto, em muitos casos, a piora da saúde.

A decisão de manter ou suspender a alimentação e a hidratação de pacientes que estão em cuidados paliativos deve ser discutida com a equipe técnica multiprofissional, com o paciente e com seus familiares 10. Em alguns casos, o próprio paciente decide não mais se alimentar, e esta postura deveria ser respeitada, do ponto de vista moral e ético, pelo médico, considerando os princípios da autonomia. Porém, nem sempre essa decisão é acatada ${ }^{41}$. Há situações em que a recusa voluntária de alimentos e água pelo paciente está relacionada à intenção de apressar a morte, em decorrência de depressão, que, se tratada, pode reverter esse quadro ${ }^{42}$. Da perspectiva ética, os princípios da autonomia, beneficência e não-maleficência apóiam os direitos do paciente em refutar ou questionar a retirada de algum tipo de terapia. Todavia, apesar das opiniões éticas e legais do assunto, alguns autores alegam que a retirada do tratamento nutricional é insustentável e deveria ser evitada 41 .

A idéia da abstinência alimentar desencadeia um sofrimento adicional para o paciente, o familiar e/ou cuidador e para os profissionais de saúde, uma vez que a progressão da doença leva à perda de peso, com impacto nas condições físicas além de conseqüências psicossociais que podem comprometer a qualidade de vida 25,43 .

Dentre as estratégias de nutrir e de hidratar, consideram-se não apenas a utilização da via oral, mas também a possibilidade da nutrição artificial por meio de cateter, adaptada aos pacientes que apresentam incapacidade total ou parcial de se alimentar oralmente 44 . Apesar de a indicação da dieta enteral ser médica, vários profissionais estão envolvidos nessa conduta, visando à melhora do suporte do paciente, o que resultará na melhora dos cuidados gerais 10,44. 
Administração de sintomas por meio da alimentação 4,10.

\begin{tabular}{|c|c|}
\hline Sintomas & Conduta \\
\hline \multirow[t]{5}{*}{ Anorexia } & Oferecer alimentos preferidos e saborosos \\
\hline & Fracionar as refeições em pequenas quantidades \\
\hline & Enriquecer o valor nutricional dos alimentos (manteiga, óleo, mel, açúcar etc.) \\
\hline & Não forçar a alimentação \\
\hline & Encorajar o desejo de alimentar-se \\
\hline \multirow[t]{3}{*}{ Saciedade precoce } & Fracionar as refeições \\
\hline & Diminuir o volume dos alimentos \\
\hline & Reduzir oferta de alimentos gordurosos e vegetais crus \\
\hline \multirow[t]{3}{*}{ Xerostomia } & Beber água freqüentemente e em pequena quantidade \\
\hline & Mascar chiclete sem açúcar \\
\hline & Preferir alimentos com molhos e caldos \\
\hline \multirow[t]{5}{*}{ Náuseas e vômitos } & Fracionar as refeições \\
\hline & Evitar odores fortes e temperos nos alimentos \\
\hline & Evitar alimentos com temperaturas extremas \\
\hline & Evitar beber líquidos durante as refeições \\
\hline & Evitar alimentos açucarados \\
\hline \multirow[t]{3}{*}{ Disgeusia } & Utilizar temperos e aromas artificiais \\
\hline & Prestar atenção na temperatura dos alimentos \\
\hline & Substituir alimentos desagradáveis por outros do mesmo valor nutricional \\
\hline \multirow{3}{*}{$\begin{array}{l}\text { Mucosite, distúrbio de mastigação } \\
\text { ou de deglutição }\end{array}$} & Evitar alimentos e bebidas irritantes (especiarias, bebidas alcoólicas, \\
\hline & alimentos duros, salgados e a ácidos) \\
\hline & Evitar alimentos quentes \\
\hline \multirow[t]{2}{*}{ Constipação } & Beber líquidos adequadamente \\
\hline & Associar diferentes tipos de fibras: hortaliças, cereais, frutas (ameixa, figo, uvas) \\
\hline \multirow[t]{2}{*}{ Diarréia } & Beber líquidos adequadamente \\
\hline & Evitar alimentos laxativos \\
\hline \multirow[t]{3}{*}{ Obstrução intestinal } & Preparar alimentos bem cozidos e pastosos \\
\hline & Evitar frutas e hortaliças cruas e com cascas, sementes, frutas oleaginosas, especiarias. \\
\hline & Introduzir alimentação artificial profilática \\
\hline \multirow[t]{2}{*}{ Hipercalcemia } & Evitar suplementos de cálcio. Manter as necessidades conforme \\
\hline & ingestão diária recomendada \\
\hline Hiponatremia & Restringir líquidos \\
\hline \multirow[t]{2}{*}{ Trombose } & Sem necessidade de restringir alimentos que contenham vitamina $\mathrm{K}$ \\
\hline & Evitar suplementos de vitamina $\mathrm{K}$ \\
\hline
\end{tabular}

Em pacientes sem possibilidade de cura, a eficácia do suporte nutricional e da hidratação ainda é controversa. Alguns estudos são realizados para avaliar o impacto dos benefícios dessas condutas na qualidade de vida dos pacientes $3,7,26,35,45,46$. Suporte nutricional é um cuidado de apoio e se insere, na situação paliativa, na responsabilidade global, objetivando manter ou recuperar o bem-estar do paciente 10 .

Em muitos casos, nem sempre o alimento promoverá o conforto e o bem-estar 10,35. Os efeitos indesejáveis das técnicas da nutrição, em especial da nutrição artificial, são, algumas vezes, causas de piora da qualidade de vida, prejudicando o objetivo real dos cuidados paliativos 3,10 . Essa contradição pode ser exemplificada em diversas situações nas quais os pacientes apresentam desde perda de autonomia para se alimentar, passando por sintomas diversos do trato gastrintestinal, até impossibilidade total de alimentação 10 .

Autores têm enfatizado a importância da natureza simbólica do alimento na provisão da nutrição e hidratação no paciente terminal 10,11,35,45. 
Existem, contudo, muitas controvérsias desse tema $3,35,46$. Na fase terminal, certos doentes podem sofrer com a alimentação oral (desconforto abdominal e náuseas), a qual eles mantêm para tranqüilizar ou agradar aos seus familiares 10 .

Nos cuidados ao fim da vida, a alimentação ainda é um assunto conflitante que envolve fatores como contradições, mitos e emoções; por isso, é importante definir as condutas conforme o desejo do paciente e da família, cuja tomada de decisão pode estar baseada na cultura 3,10,24 . Decidir sobre a alimentação e hidratação nesse momento é muito complicado, pois são temas polemizados há décadas 10,35, e os benefícios do suporte nutricional ou da hidratação permanecem questionados 3,10,35. A quantidade ideal de calorias ou nutrientes e o volume adequado de líquidos ainda são questões em debate perenes 35 .

Refletir sobre questões de alimentação relacionadas à finitude sempre provoca polêmicas e opiniões discrepantes entre profissionais de saúde, familiares e o próprio paciente 14 . Em algumas situações, o paciente não se encontra apto a tomar decisões, ficando dependente da decisão dos familiares acerca do plano de cuidados propostos 15 .

Negar medidas fúteis de tratamento, que visam a prolongar a vida sem melhora do quadro clínico, ainda é controverso entre o Ocidente e a Ásia. Na Coréia, por exemplo, onde se considera o tempo de vida extremamente valioso, defendese que se deva prolongá-lo tanto quanto possível, ainda que usando medidas fúteis disponíveis. Nessa cultura, opta-se por omitir do paciente tanto a doença, quanto o seu estádio, inviabilizando o exercício da autonomia 14.

Em contraste, no Ocidente, valoriza-se a autonomia dos pacientes na tomada de decisão do plano de cuidados, uma vez que eles, de modo geral, preferem que se revele a verdade. Essa autonomia evidencia a natureza da diferença cultural entre o Ocidente e a Ásia 14. No Brasil, tendo como base o Código de Ética Médica, o paciente tem o direito de consentir ou recusar de forma livre, voluntária e esclarecida, os tratamentos aos quais será submetido 47 .

O emblemático caso americano "Terri Schiavo", por exemplo, apesar de discutir os mesmos conflitos, apresenta um contexto clínico de estado vegetativo permanente, provocado por uma parada cardíaca com prejuízo total da função do córtex cerebral 48,49 , gerando, entre outras, as se- guintes conseqüências: alternância de ciclos sono/vigília, ausência aparente da consciência de si e do ambiente circunstante e falta de respostas aos estímulos ambientais 21 .

Na época, esse caso provocou uma repercussão mundial, por causa de distorções de grupos de interesse e de exagero da mídia 48 . Muitos pesquisadores e profissionais paliativistas, os quais acreditam que, algumas vezes, a morte por desnutrição e desidratação não é dolorosa nem sofrida - embora sem evidência na literatura -, foram a público para tranqüilizar a população, asseverando que a morte da paciente foi um processo humano e indolor 49 .

É importante ressaltar que o estado vegetativo é nitidamente distinto da fase terminal de uma doença, definido por muitos autores como o período de vida prolongado até a morte. No caso do câncer, esse período pode durar dias ou até meses, apesar dos sintomas preditivos da morte 10,50.

\section{Conclusão}

A preocupação em poder alimentar o paciente com estádio avançado de câncer, bem como a forma e as estratégias de procedimento ainda são causas de discussão entre os profissionais de saúde. Além do controle dos sinais e sintomas, existe a necessidade de se conhecerem os hábitos alimentares dessa população. Embora pouco divulgada na literatura científica, essa abordagem é relevante para um aconselhamento nutricional mais efetivo que possa, de alguma maneira, refletir na melhora da qualidade de vida 28 .

O estado de saúde é influenciado pelos aspectos nutricionais. Em virtude de sua relevante contribuição para a qualidade de vida, o cuidado nutricional deve estar integrado aos cuidados oncológicos globais. Trata-se de uma intervenção que demanda esforço e dedicação, devendo ser realizada por profissionais muito conscientes $4,7,9,10$.

Logo, o profissional nutricionista, sobretudo nesse contexto, tem um papel técnico de grande valia. A sensibilidade e criatividade farão a diferença durante a avaliação e o aconselhamento nutricionais. Deve-se respeitar o paciente e considerar os recursos terapêuticos para o controle de sintomas, valorizando os alimentos preferenciais, a adequação da dieta e o desejo do próprio paciente por alimentos. 


\section{Resumo}

O câncer vem se destacando entre as doenças crônicas, sendo a segunda causa de morte em países desenvolvidos. Os cuidados paliativos se propõem a oferecer cuidados de suporte global, visando ao controle de sintomas e à melhora da qualidade de vida dos pacientes e de seus familiares. A nutrição é uma ferramenta importante nos cuidados paliativos, podendo ajudar o paciente nos aspectos físico, psicológico e social, além de promover o bem-estar e a qualidade de vida. Entretanto, a nutrição raramente poderá cumprir seu papel de recuperar e garantir o estado nutricional. Nesse momento, a intervenção do nutricionista é considerar as necessidades do indivíduo, preferências e hábitos alimentares, fundamentais tanto para o controle dos sintomas, quanto para garantir satisfação e conforto. A impossibilidade do exercício convencional das condutas já estabelecidas e o desenvolvimento de uma nova percepção do doente muitas vezes produzem dilemas para o profissional nutricionista.

Cuidados Paliativos; Qualidade de Vida; Neoplasias; Bioética; Nutrição em Saúde Pública

\section{Referências}

1. World Health Organization. Preventing chronic diseases: a vital investment. Geneva: World Health Organization; 2005.

2. World Health Organization. National cancer control programmes: policies and managerial guidelines. Geneva: World Health Organization; 2002.

3. Moynihan T, Kelly DG, Fisch MJ. To feed or not to feed: is that the right question? J Clin Oncol 2005; 23:6256-9.

4. Elliott L, Molseed LL, McCallum PD, Grant B. The clinical guide to oncology nutrition. 2nd Ed. Chicago: American Dietetic Association; 2006.

5. Jocham HR, Dassen T, Widdershoven G, Halfens R. Quality of life in palliative care cancer patients: a literature review. J Clin Nurs 2006; 15:1188-95.

6. World Health Organization. Study protocol for the World Health Organization project to develop a Quality of Life assessment instrument (WHOQOL). Qual Life Res 1993; 2:153-9.

7. Caro MMM, Laviano A, Pichard C. Nutritional intervention and quality of life in adult oncology patients. Clin Nutr 2007; 26:289-301.

8. Commission directive 1999/21/EC of 25 March 1999 on dietary foods for special medical purposes. http://www.idace.org/legislation/fsmps/ Dir\%2099-21\%20FSMPs.pdf (acessado em 20/Fev/ 2008).

\section{Colaboradores}

M. O. Benarroz e G. B. D. Faillace redigiram o trabalho. L. A. Barbosa revisou o artigo.

\section{Agradecimentos}

Agradecemos a preciosa revisão do texto pelo Prof. Alexandre Florêncio.
9. Cid-Conde L, Fernández-López T, Neira-Blanco P, Arias-Delgado J, Varela-Correa JJ, Gómez-Lorenzo FF. Prevalencia de desnutrición en pacientes con neoplasia digestiva previa cirugía. Nutr Hosp 2008; 23:46-53.

10. Bachmann P, Marti-Massoud C, Blanc-Vincent MP, Desport JC, Colomb V, Dieu L, et al. Standards, options et recommandations: nutrition en situation palliative ou terminale de l'adulte porteur de cancer évolutif. Bull Cancer 2001; 88:985-1006.

11. Hopkins K. Food for life, love and hope: an exemplar of the philosophy of palliative care in action. Proc Nutr Soc 2004; 63:427-9.

12. Silva RCF, Hortale VA. Cuidados paliativos oncológicos: elementos para o debate de diretrizes nesta área. Cad Saúde Pública 2006; 22:2055-66.

13. Silva CHD. A moralidade dos cuidados paliativos. Rev Bras Cancerol 2004; 50:330-3.

14. Oh DY, Kim JE, Lee CH, Lim JS, Jung KH, Heo DS, et al. Discrepancies among patients, family members, and physicians in Korea in terms of values regarding the withholding of treatment from patients with terminal malignancies. Cancer 2004; 100:1961-6.

15. Floriani CA, Schramm FR. Cuidados paliativos: interfaces, conflitos e necessidades. Ciênc Saúde Coletiva 2008; 13 Suppl 2:2123-32. 
16. Manckoundia P, Mischis-Troussard C, Ramanantsoa M, Blettery B, François-Pursell I, Martin-Pfitzenmeyer I, et al. Les soins palliatifs en gériatrie: étude rétrospective de 40 cas. Rev Med Interne 2005; 26:851-7.

17. Goldim JR. Bioética: origens e complexidade. Rev HCPA \& Fac Med Univ Fed Rio Gd do Sul 2006; 26:86-92.

18. Boccatto M. A importância da bioética. Genética na Escola 2007; 4:11-4.

19. Silva MF, Fernandes MFP. A ética do processo ante o gerenciamento de enfermagem em cuidado paliativo. Mundo Saúde 2006; 30:318-25.

20. Ferrai CMM, Silva L, Paganine MC, Padilha KG, Gandolpho MA. Uma leitura bioética sobre cuidados paliativos: caracterização da produção científica sobre o tema. Mundo Saúde 2008; 2:99-104.

21. Pessini L. Dignidade humana nos limites da vida: reflexões éticas a partir do caso de Terri Schiavo. Bioética 2005; 13:65-76.

22. Araújo MMT, Silva MJP. A Comunicação com o paciente em cuidados paliativos: valorizando a alegria e o otimismo. Rev Esc Enferm USP 2007; 41:668-74.

23. Azevedo EES. Ensino de bioética: um desafio transdisciplinar. Interface Comun Saúde Educ 1998; 2:127-38.

24. McMahon MM, Hurley DL, Kamath SP. Medical and ethical aspects of long-term enteral tube feeding. Mayo Clin Proc 2005; 80:1461-76.

25. Hopkinson J, Wright D, Corner J. Exploring the experience of weight loss in people with advanced cancer. J Adv Nurs 2006; 54:304-12.

26. Laviano A, Mequide MM, Inui A, Muscaritoli M, Rossi-Fanelli F. Therapy insight: cancer anorexiacachexia syndrome-when all you can eat is yourself. Nat Clin Pract Oncol 2005; 2:158-65.

27. Von Roenn JH, Paice JA. Control of common, nonpain cancer symptoms. Semin Oncol 2005; 32:20010.

28. Hutton JL, Matin L, Field JC, Wismer WV, Bruera ED, Watanabe SM, et al. Dietary patterns in patients with advanced cancer: implications for anorexiacachexia therapy. Am J Clin Nutr 2006; 84:1163-70.

29. Dy SM, Lorenz KA, Naeim A, Sanati H, Walling A, Asch SM. Evidence-based recommendations for cancer fatigue, anorexia, depression, and dyspnea. Clin Oncol 2008; 26:3886-95.

30. Folke S, Fridlund B, Paulsson G. Views of xerostomia among health care professionals: a qualitative study. J Clin Nurs 2008; 18:1-8.

31. Hutton JL, Baracos VE, Wismer WV. Chemosensory dysfunction is a primary factor in the evolution of declining nutritional status and quality of life in patients with advanced cancer. J Pain Symptom Manage 2007; 33:156-65.

32. Feio M, Sapeta P. Xerostomia em cuidados paliativos. Acta Med Port 2005; 18:459-66.

33. Sloane PD, Ivey J, Helton M, Barrick AL, Cerna A. Nutritional issues in long-term care. J Am Med Dir Assoc 2008; 9:476-85.

34. Hopkinson JB, Wright DN, McDonald JW, Corner JL. The prevalence of concern about weight loss and change in eating habits in people with advanced cancer. J Pain Symptom Manage 2006; 32:322-31.
35. Oh DY, Kim JH, Lee SH, Kim DW, Im SA, Kim TY, et al. Artificial nutrition and hydration in terminal cancer patients: the real and the ideal. Support Care Cancer 2007; 15:631-6.

36. Dormann A J. Endoscopic palliation and nutritional support in advanced gastric cancer. Dig Dis 2004; 22:351-9.

37. Elia M, Van Bokhorst-de van der Schueren MA, Garvey J, Goedhart A, Lundholm K, Nitenberg G, et al. Enteral (oral or tube administration) nutritional support and eicosapentaenoic acid in patients with cancer: a systematic review. Int J Oncol 2006; 28:5-23.

38. Instituto Nacional de Câncer. Cuidados paliativos oncológicos: controle da dor. http://bvsms.saude. gov.br/bvs/publicacoes/inca/manual_dor.pdf (acessado em 31/Jul/2008).

39. Boström B, Sandh M, Lundberg D, Fridlund B. A comparison of pain and health-related quality of life between two groups of cancer patients with differing average levels of pain. J Clin Nurs 2003; 12:726-35.

40. Silva MEMP, Paton I, Trigo M. Vida saudável: nutrição, nutrientes, alimentos, saúde. São Paulo: Faculdade de Saúde Pública, Universidade de São Paulo; 1999.

41. Farber NJ, Simpson P, Salam T, Collier VU, Weiner J, Boyer EG. Physicians' decisions to withhold and withdraw life-sustaining treatment. Arch Intern Med 2006; 166:560-4.

42. Ganzini L, Goy ER, Miller LL, Harvath TA, Jackson A, Delorit MA. Nurses' experiences with hospice patients who refuse food and fluids to hasten death. N Engl J Med 2003; 349:359-65.

43. Poole K, Froggatt K. Loss of weight and loss of appetite in advanced cancer: a problem for the patient, the carer, or the health professional? Palliat Med 2002; 16:499-506.

44. Tran M, Raynard B, Bataillard A, Duguet A, Garabige $\mathrm{V}$, Lallemand $\mathrm{Y}$, et al. Bonnes pratiques pour la prise en charge diététique en cancérologie: la nutrition entérale (rapport abrégé). Bull Cancer 2006; 93:715-22.

45. Chiu TY, Hu WY, Chuanh RB, Chen CY. Nutrition and hydration for terminal cancer patients in Taiwan. Support Care Cancer 2002; 10:630-6.

46. Van der Riet P, Brooks D, Ashby M. Nutrition and hydration at the end of life: pilot study of a palliative care experience. J Law Med 2006; 14:182-98.

47. Conselho Federal de Medicina. Código de ética médica. Resolução CFM no. 1.246/88. http://www. portalmedico.org.br (acessado em 12/Jan/2009).

48. Quill TE. Terri Schiavo: a tragedy compounded. N Engl J Med 2005; 352:1630-3.

49. Breitbart W. What can we learn from the death of Terri Schiavo? Palliat Support Care 2005; 3:1-3.

50. Vigano A, Bruera E, Jhangri GS, Newman SC, Fields AL, Suarez-Almazor ME. Clinical survival predictors in patients with advanced cancer. Arch Intern Med 2000; 160:861-8.

Recebido em 04/Nov/2008

Versão final reapresentada em 16/Abr/2009 Aprovado em 15/Mai/2009 